\title{
Correction to: Trends and risk factors for 30-day readmissions in patients with acute cholangitis: analysis from the national readmission database
}

\author{
Malav P. Parikh ${ }^{1}$. Rajat Garg ${ }^{1}$ - Vibhu Chittajallu ${ }^{1}$. Niyati Gupta ${ }^{1}$ - Shashank Sarvepalli ${ }^{1}$ Rocio Lopez $^{3}$. \\ Prashanthi N. Thota ${ }^{2} \cdot$ Hassan Siddiki $^{2} \cdot$ Amit Bhatt $^{2} \cdot$ Prabhleen Chahal $^{2} \cdot$ Sunguk Jang ${ }^{2} \cdot$ Tyler Stevens $^{2}$. \\ John Vargo ${ }^{2}$. Arthur McCullough ${ }^{2} \cdot$ Madhusudhan R. Sanaka $^{2,4}$
}

Published online: 25 January 2022

(c) Springer Science+Business Media, LLC, part of Springer Nature 2022

\section{Surgical Endoscopy (2021) 35:223-231 https://doi.org/10.1007/s00464-020-07384-z}

This article was updated to include the funding information for Shashank Sarvepalli.

Publisher's Note Springer Nature remains neutral with regard to jurisdictional claims in published maps and institutional affiliations.

The original article can be found online at https://doi.org/10.1007/ s00464-020-07384-z.

Madhusudhan R. Sanaka

sanakam@ccf.org

Malav P. Parikh

malavparikh88@yahoo.com

Rajat Garg

drgargrajat@gmail.com

Niyati Gupta

niyati.gupta@yahoo.com

Shashank Sarvepalli

Shashank.sarvepalli@gmail.com

Rocio Lopez

lopezr@ccf.org

Prashanthi N. Thota

thotap@ccf.org

Amit Bhatt

bhatta3@ccf.org

Prabhleen Chahal

chahalp@ccf.org
Sunguk Jang

jangs@ccf.org

Tyler Stevens

stevent@ccf.org

John Vargo

vargoj@ccf.org

Arthur McCullough

mcculla@ccf.org

1 Department of Hospital Medicine, Cleveland Clinic Foundation, Cleveland, USA

2 Department of Gastroenterology and Hepatology, Cleveland Clinic Foundation, Cleveland, USA

3 Department of Biostatistics and Quantitative Health Sciences, Cleveland Clinic Foundation, Cleveland, USA

4 Department of Gastroenterolgy, Cleveland Clinic Foundation, Desk Q39500 Euclid Avenue, Cleveland, OH 44195, USA 